\title{
The proposed legislation on termination of pregnancy does not protect women or children in Malawi and is not fit for the intended purpose: Christian Medical and Dental Fellowship ${ }^{i}$ position
}

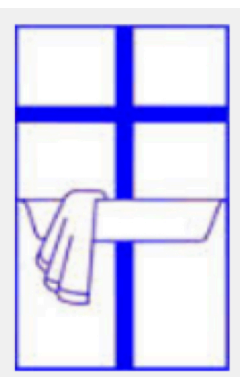

Beatrice Mwagomba $a^{1,2,3}$, M. Jane Bates ${ }^{2,3,7}$, Reynier G. Ter Haar ${ }^{2,4}$, Martha Masamba $^{2,5}$, Sekeleghe Kayuni ${ }^{2,6,}$, Isaac Chirwa ${ }^{2,8}$

1. Ministry of Health, Lilongwe, Malawi

2. Christian Medical and Dental Fellowship, Malawi

3. College of Medicine, University of Malawi, Blantyre, Malawi

4. Nkhoma Hospital, Nkhoma, Malawi

5. Stellenbosch University, Stellenbosch, South Africa

6. MASM Medi Clinics Limited, Malawi

7. Liverpool School of Tropical Medicine, Liverpool, United Kingdom

8. Asamala Health Services, Lilongwe, Malawi

Correspondence: Dr Jane Bates (mjanebates@gmail.com)

This legislation represents a significant move towards liberalisation of abortion in Malawi. Those supporting the legislation have founded their arguments mainly on the premise that it will protect women and reduce maternal mortality. We join with them to express our concern with the high number of maternal deaths in the country and firmly assert our commitment and role to ensure that no woman dies from pregnancy-related complications. Aside from the maternal mortality arguments, many of those supporting this legislation take a "pro-choice" position, insisting that the rights of a woman who is pregnant supersede any rights of her unborn child, sidelining both male (parental) responsibility and the responsibility of society to take charge of the wider societal issues raised by "unwanted" pregnancies.

We assert that the bill does not mitigate the problem of maternal mortality. Detailed study of the proposed clauses in this bill leads us to conclude that more lives could be lost if the bill was passed into law. We argue that:

- the emphasis on maternal mortality reduction has been misleading

- Malawi has inadequate regulatory frameworks to verify police reports and ensure that consent is informed

- Malawi has insufficient capacity in expertise to ensure the accurate diagnosis of non-survivable congenital abnormality

- the proposed legislation provides inadequate protection against abortion on demand, in the wording of clause 3-1-b, which cites the prevention of "injury to the physical or mental health of a pregnant woman" as grounds for termination of a pregnancy

- the prevailing culture of tolerance towards gender based and intimate partner violence leaves women and girls with little choice in areas of reproductive health

The emphasis on maternal mortality reduction has been misleading

The recentdeclinein maternal mortality in Malawihas occurred despite having the current "restrictive" law. ${ }^{1}$ Authors agree that maternal mortality is largely preventable - the consensus outside the abortion debate points towards inadequate health systems and poor access to family planning being the most significant contributors to maternal mortality. ${ }^{2}$ The World Health Organization (WHO) estimates that roughly 13\% of maternal deaths may be accounted for by unsafe abortions, but the validity of studies contributing to this estimate have been questioned. ${ }^{3}$ Liberalisation of the abortion laws is not the most effective way to reduce maternal mortality. At Nkhoma Hospital in rural Lilongwe, maternal mortality rates fell from 1518/100,000 to 109/100,000 between 2008 and 2015 by improving obstetric care within the confines of the current restrictive abortion law. (Nkhoma HMIS data, 2008 to 2015). An in-depth review of 58 maternal deaths at the same hospital, from 2007 to 2011, showed that only 3 maternal deaths $(5.2 \%)$ were related to abortion complications. ${ }^{4}$ Most patients experienced a delay in seeking care ( $\mathrm{n}=37 ; 63.8 \%)$, a transport delay $(\mathrm{n}=43 ; 74.1 \%)$, or a delay in receiving adequate care $(\mathrm{n}=34 ; 58.6 \%$ ). These common and important factors need to be addressed as a matter of priority if a country wants to improve maternal outcomes.

Similarly, a study in Mexico found that differences in maternal deaths between states with restrictive and permissive abortion laws were not explained by abortion legislation, but by other factors, such as literacy rates among women, maternal healthcare, access to clean water, proper sanitation facilities, fertility rates, and the level of violence against women. Loosening abortion restrictions did not produce a drop in maternal mortality rates. ${ }^{5}$ ) The study suggests that developing countries do not need to relax abortion laws to reduce maternal mortality rates.

\section{Malawi has inadequate regulatory frameworks to verify that police reports are accurate and ensure that consent is informed}

The suggested rape clause is open to abuse since it is still possible, in certain cases, to acquire police reports for a fee. Consent processes remain poorly developed (a situation affecting a variety of procedures in the medical field), with very little required from health workers to ensure that women and girls fully understand the implications of undergoing an abortion. "Counselling", in Malawi, is a term that is MMJ VOL 29 (1): March 2017 
understood to mean "giving advice" rather than listening to and supporting a person through issues they face, and many health workers in Malawi are not skilled in patient-centred counselling. This brings about the risk that vulnerable women and girls will be advised to a certain course of action without fully understanding either what is happening to them, or the potential physical and psychosocial consequences (including the risks of bleeding and infection).

\section{Malawi has insufficient expertise to ensure the necessary capacity for the accurate diagnosis of non-survivable congenital abnormality}

The legislation proposes that any certified health provider may perform an abortion in the situation of a congenital malformation that affects foetal viability or compatibility with life. This clause refers to very few conditions, which would be detected by the use of ultrasound scanning. Initially, the proposed legislation had required subjective evidence (in the "opinion" of the health worker, and "in good faith"); this has been changed to require ultrasound scanning. Malawi has limited health structures, resources, and staffing in most rural areas, where almost $75 \%$ of Malawians are located. Most facilities are run by only a single nurse, who is overstretched and supported by unskilled staff. There is every possibility that abortion would be recommended for a variety of conditions (for example, hydrocephalus, spina bifida, and Down's syndrome), which are survivable and amenable to medical intervention.

CMDF recommends that this clause should be removed.

\section{There is inadequate protection against abortion on demand in the wording of clause 3-1-b}

This clause, which cites prevention of "injury to the physical or mental health of a pregnant woman" as grounds for termination of a pregnancy, is not supported by current data from the scientific literature. While there is an increase in mental illness resulting from carrying an unwanted pregnancy, this risk is the same whether the woman has an abortion or goes on to give birth. ${ }^{6}$ Additionally, having an abortion does not reduce the risk of mental illness.

While we recognise that the wording in the proposed bill is not as liberal as the South African Choice on Termination of Pregnancy Act 1996, ${ }^{6}$ the inclusion of this clause (3-1-b) is of grave concern to us, as it has led other countries to effectively practice abortion on demand, which the Malawi Special Law Commission on the Review of the Law on Abortion have specifically stated they want to avoid. A United Kingdom government report states that $98 \%$ of the 2 million abortions performed in the UK, from 2005 to 2015, took place under a clause with similar wording to that in clause 3-1-b of the proposed Malawi Termination of Pregnancy Bill. ${ }^{7}$ The Special Law Commission states that the mother should be at risk of being a "physical or mental wreck" according to assessment by a psychiatrist. Neither of these statements is appropriate or realistic. There are no medical or psychiatric criteria to define the terms "physical or mental wreck" included in the Special Law Commission wording. Nationally, psychiatric services are grossly insufficient to assess these criteria. This leaves the clause wide open to individual interpretation-a licence to practise abortion on demand for those who see fit.

\section{The prevailing culture of tolerance towards gender-based and intimate partner violence leaves women and girls with little choice in areas of reproductive health}

Gender-based violence in Malawi is common and accepted. National surveys report that $25 \%$ of women report experiencing sexual violence and $38.1 \%$ of girls aged 12 to 19 years report that their first sexual experience was against their will. ${ }^{8}$ In this debate, access to abortion is frequently proposed as the solution to this problem, giving women "rights", yet nothing is heard about male responsibility, and the rights of the unborn are dismissed as of little consequence. Men are complicit in every pregnancy. Practises involving coercive sexual intercourse were cited as a reason for procuring abortion in the strategic review of unsafe abortion by Jackson et al. published in 2011. ' Traditional practises and power dynamics often force women and girls into positions of extreme vulnerability in terms of negotiating family planning and sexual intercourse itself. The resulting unwanted pregnancies leave women and girls with the consequences, knowledge, and experience all their lives, while men seemingly have nothing to lose and bear no responsibility for their actions. This bill upholds the current status quo of male domination in sexual relationships, with inadequate assessment, consent, and management of abortion and its complications being just another form of gender-based violence against women and girls, who would once again pay the price for "man-made" choices.

There is no provision in the proposed legislation for society to protect its most vulnerable. Civil society (including communities of faith) needs to strengthen and implement messages and activities that reduce gender-based violence. Women and girls should be equipped with skills and empowered to negotiate in areas of sexual and reproductive health. Women should be supported to carry pregnancies to term and place their children for adoption if they choose. Malawi is founded on principles of Ubuntu: this should be upheld in reproductive health to ensure the right to life for all.

\section{Conclusions}

While welcoming the clear process of determination of suitable laws and involvement of all sectors of the Republic of Malawi, CMDF does not support the current proposed legislation on termination of pregnancy. We recommend that the law continues to support abortion only if the life of the mother is at risk and call for the removal of the other clauses. To reduce maternal mortality, CMDF calls for and works towards access to essential health services, including the urgent need for improved access to family planning and more attention to (and management of) the delays associated with poor pregnancy outcomes. Reduction in violence against women and girls and better provision of adoption services are required. Such measures provide a safe and ethical strategy to reduce the incidence of unsafe abortion, benefitting all sectors including the men, women, and children (including those yet to be born) of Malawi.

[i] CMDF Malawi is a national inter-denominational fellowship of Christian doctors and dental surgeons. Since its beginning in 1998 it has been bringing together Christian medical and dental professionals for mutual encouragement and fellowship 'to spur each other on toward love and good deeds' by optimising the gifts and resources of our Christian medical and dental fraternity, to sharpen each other in our faith and walk as Christian doctors and dental surgeons. CMDF Malawi is a member of the International Christian Medical and Dental Fellowship (ICMDA), a global movement of over 80 countries. 


\section{References}

1. Colbourn T, Lewycka S, Nambiar B, Anwar I, Phoya A, Mhango C. Maternal mortality in Malawi, 1977-2012. BMJ Open. 2013 Jan 12;3(12):e004150.

2. Bazile J, Rigodon J, Berman L, Boulanger VM, Maistrellis E, Kausiwa $\mathrm{P}$, et al. Intergenerational impacts of maternal mortality: Qualitative findings from rural Malawi. Reprod Health. 2015;12(1):S1. 3. Gerdts C, Vohra D, Ahern J. Measuring Unsafe Abortion-Related Mortality: A Systematic Review of the Existing Methods. PLoS ONE [Internet]. 2013 Jan 14 [cited 2016 Nov 13];8(1). Available from: http:// www.ncbi.nlm.nih.gov/pmc/articles/PMC3544771/

4. Vink NM, de Jonge HCC, Ter Haar R, Chizimba EM, Stekelenburg J. Maternal death reviews at a rural hospital in Malawi. Int J Gynaecol Obstet Off Organ Int Fed Gynaecol Obstet. 2013 Jan;120(1):74-7.

5. Koch E, Chireau M, Pliego F, Stanford J, Haddad S, Calhoun B, et al. Abortion legislation, maternal healthcare, fertility, female literacy, sanitation, violence against women and maternal deaths: a natural experiment in 32 Mexican states. BMJ Open. 2015 Jan 2;5(2):e006013.
6. Choice on Termination of Pregnancy Act 92 of 1996 - Act92of1996. pdf [Internet]. [cited 2016 Nov 15]. Available from: http://www.gov.za/ sites/www.gov.za/files/Act92of1996.pdf

7. Abortion statistics, England and Wales: 2015 - Statistical data sets GOV.UK [Internet]. [cited 2016 Nov 15]. Available from: https://www. gov.uk/government/statistical-data-sets/abortion-statistics-englandand-wales-2015

8. Gender-based Violence in Malawi: A Literature Review to Inform the National Response - 436_FINALHPPMalawiGBVLiteratureReview .pdf [Internet]. [cited 2016 Nov 15]. Available from: http:// www.healthpolicyproject.com/pubs/436_FINALHPPMalawi GBVLiteratureReview.pdf

9. Jackson E, Johnson BR, Gebreselassie H, Kangaude GD, Mhango C. A strategic assessment of unsafe abortion in Malawi. Reprod Health Matters. 2011 May;19(37):133-43. 